Como citar este artículo:

Flórez-Durango, A.M., González-Zuluaga, V.M., Londoño-Herrera, J.A., Zapata-García, T.V. y Montoya-Zuluaga, P.A. (2016). Caracterización de la memoria en niños con trastorno del espectro autista sin déficit intelectual. Una aproximación para el acompañamiento de los procesos de enseñanza-aprendizaje. Revista Eleuthera, 14, 11-29. DOI: $10.17151 /$ eleu.2016.14.2.

\title{
CARACTERIZACIÓN DE LA MEMORIA EN NIÑOS CON TRASTORNO DEL ESPECTRO AUTISTA SIN DÉFICIT INTELECTUAL. UNA APROXIMACIÓN PARA EL ACOMPAÑAMIENTO DE LOS PROCESOS DE ENSEÑANZA- APRENDIZAJE*
}

\section{CHARACTERIZATION OF MEMORY IN CHILDREN WITH AUTISM SPECTRUM DISORDER WITHOUT INTELLECTUAL DEFICIT. AN APPROACH TO ACCOMPANYING THE TEACHING-LEARNING PROCESSES}

\author{
Ana María Flórez-Durango** \\ Verónica Marcela González-Zuluaga*** \\ Jennifer Andrea Londoño-Herrera**** \\ Tatiana Vanessa Zapata-García***** \\ Paula Andrea Montoya-Zuluaga******
}

\section{Resumen}

Objetivo. El propósito del presente artículo de revisión es identificar y caracterizar el funcionamiento de la memoria en niños con Trastorno del Espectro Autista sin déficit intelectual. Metodología. Se realizó una revisión en diferentes bases de datos; se estimó como criterio de inclusión artículos resultado de investigación entre 2009 y 2015, cuya muestra fueran niños y adolescentes. Resultados. Se encontró que las personas con autismo presentan compromisos en la memoria de trabajo, memoria episódica y memoria procedimental. Conclusiones. Reconocer el funcionamiento de los diferentes sistemas de memoria en el Trastorno del Espectro Autista posibilitará al maestro generar estrategias en el aula de clase, que faciliten los procesos de enseñanza-aprendizaje, lo que revertirá en un desarrollo humano integral.

Palabras clave: memoria de trabajo, memoria episódica, memoria procedimental, memoria fuente, procesos de memoria, autismo sin déficit intelectual.

\footnotetext{
*Artículo de revisión producto del proyecto de investigación "Estandarización del Test de Memoria y Aprendizaje (TOMAL) en niños de 5 años de edad del municipio de Medellín”. Investigadora principal Paula Andrea Montoya Zuluaga. Grupo de investigación Neurociencias Básicas y Aplicadas, Línea de Investigación - Neuropsicopedagogía. Financiación: Universidad Católica Luis Amigó y Quipux Innova (R:0408)

${ }^{* *}$ Universidad Católica Luis Amigó. Medellín, Colombia. E-mail: anaflorezdurango@gmail.com.

(D) orcid.org/0000-0001- 5362-5256.

*** Universidad Católica Luis Amigó. Medellín, Colombia. E-mail: vero.gonza1519@gmail.com.

(D) orcid.org/0000-0002- 7658-4853.

***** Universidad Católica Luis Amigó. Medellín, Colombia. E-mail: jenniferandrea18@hotmail.com.

(D) orcid.org/0000-0003-4446-554x.

****** Universidad Católica Luis Amigó. Medellín, Colombia. E-mail: tatiana.zapataga@hotmail.com.

(D) orcid.org/0000-0002-5716-639x.

******* Universidad Católica Luis Amigó. Medellín, Colombia. E-mail: paula.montoyazu@amigo.edu.com.

(D) orcid.org/0000-0001-5871-1727.
} 
Caracterización de la memoria en niños con trastorno del espectro autista sin déficit intelectual...

\begin{abstract}
Objective. The purpose of this review article is to identify and characterize the memory functioning in children with Autism Spectrum Disorder without intellectual deficit. Methodology. A review in different databases was carried out. Articles resulting from research between 2009 and 2015, whose sample were children and adolescents, were considered as inclusion criteria. Results. It was found that people with autism show impairments in working memory, episodic memory and procedural memory. Conclusions. Recognizing the functioning of the different memory systems in autism spectrum disorder will make possible for the teacher to generate strategies to facilitate teachinglearning processes in the classroom, which will revert to a comprehensive human development.
\end{abstract}

Key words: working memory, episodic memory, procedural memory, source memory, memory processes, autism spectrum disorder without accompanying intellectual impairment.

\title{
Introducción
}

En el marco actual de diagnóstico según el DSM-5 (Manual diagnóstico y estadístico de los trastornos mentales- APA, 2014), el Trastorno del Espectro Autista (TEA) se caracteriza por deficiencias persistentes en la comunicación e interacción social y patrones restrictivos y repetitivos de comportamiento, intereses o actividades, los cuales deben estar presentes en las primeras fases del desarrollo y causar un deterioro clínicamente significativo en lo social, laboral u otras áreas importantes del funcionamiento habitual y no se explican por una discapacidad intelectual o por retraso global del desarrollo. De acuerdo con lo plasmado en el DSM-IV-TR (2002), el TEA recibía la denominación de trastorno generalizado del desarrollo, contemplando bajo esta entidad nosológica el trastorno autista, trastorno de Rett, trastorno desintegrativo infantil, trastorno de asperger y trastorno generalizado del desarrollo no especificado. Actualmente, son clasificados bajo el término de TEA, en el que los síntomas varían según la gravedad, el nivel de desarrollo y la edad cronológica. En este sentido, los criterios diagnósticos para el trastorno autista, según el DSM-IV-TR (2002), eran caracterizados por ser más explícitos y detallados; en la actual versión, dichos criterios son establecidos desde un continuum.

Según el DSM-5 (2014), la prevalencia de dicho trastorno abarca aproximadamente el 1\% de la población, de la cual se diagnostica cuatro veces más frecuentemente en el sexo masculino que en el femenino. Además, la probabilidad de que un niño sea diagnosticado aumentó en más del 20\% de 2006 a 2008 .

Por su parte, la memoria, según Baddeley (1999), es un sistema cognitivo complejo ya que sus múltiples componentes hacen que no se comprenda como "una sola entidad, sino que, 
más bien, consiste en una serie de sistemas diferentes que tienen en común la capacidad para almacenar información” (p. 7), así como también la recuperación de la misma. Dicha información es obtenida mediante los sentidos, siendo la memoria de cierto modo un registro de percepciones en el que confluyen múltiples dominios de actividad mental. Por esto, cuando se habla de memoria, se hace referencia a un sistema compuesto por subsistemas diferenciados entre sí pero que a la vez se interrelacionan, lo cual, según Boucher, Mayes \& Bigham (2012), permite responder de manera específica frente a las demandas del medio.

El funcionamiento de la memoria en niños conTEA ha sido objeto de múltiples investigaciones a lo largo del tiempo (Schuhand Eigsti, 2012; Andersen, Hovik, Skogli, Egeland \& Øie, 2013; Jiang, Capistrano \& Palm, 2014; Goddard, Dritschel \& Howlin, 2014; Terret, Rendell, Saunders, Henry, Bailey \& Altgassen, 2013; Zalla, Labruyere \& Georgieff, 2013; Cornett, Miora, Fass \& Dixon, 2013; Bigham, Boucher, Mayes \& Anns, 2010; Lelii \& Wellman, 2012 y Lind \& Bowler, 2009; Brenner Shih, Colich, Sugar, Bearden \& Dapretto, 2014; Maister \& PlaistedGrant, 2011; Gil, Chambres, Hyvert, Fanget \& Droit-Volet, 2012; Altgassen, Williams, Bölte \& Kliege, 2009; Southwick, Bigler, Froehlich, DuBray, Alexander, Lange \& Lainhart, 2011; Wojcik, Moulin \& Souchay, 2013; Brezis, Galili, Wong \& Piggot, 2014; Erdodi, LajinessO’Neill \& Schmitt, 2013), en especial con relación al autismo de alto funcionamiento (AAF), denominado actualmente como TEA sin déficit intelectual acompañante. La variabilidad de estos términos depende de la versión del DSM en que se basaron los autores de las diversas investigaciones para realizar el estudio respectivo (AAF- DSM-IV, 2002; TEA sin déficit intelectual acompañante - DSM-5, 2014).

Dentro de los hallazgos más relevantes en las investigaciones con sujetos con diagnóstico de TEA, cabe precisar que se han encontrado compromisos en la memoria episódica (ME), en tanto se les dificulta la codificación (Southwick et al. 2011) y la recuperación (Brezis et al. 2014) de información autobiográfica. Así mismo, se refieren compromisos en la memoria de trabajo (MT), algunos hallazgos muestran déficit globales (Schuh et al., 2012; Alloway \& Alloway, 2010), mientras que otros, solo afirman compromisos en el buffer fonológico y agenda espacial (Andersen et al., 2013; Jiang et al., 2014). Adicionalmente, se afirma que los niños con TEA tienen compromisos en utilizar estrategias para acciones y pensamientos futuros, lo que lleva a algunos autores a afirmar que los niños con este diagnóstico tienen dificultades en la memoria prospectiva (MP) basada en el tiempo (Altgassen et al., 2009; Maister et al., 2011). Finalmente, los hallazgos investigativos muestran que el sistema de memoria semántico se encuentran intacto, lo que explica la habilidad que tienen los niños que hacen parte de este grupo poblacional no solo a codificar, sino a organizar, almacenar y recuperar información que hace parte de la enciclopedia mental o el conocimiento acontextual (Lind et al., 2009).

Específicamente, la ME ha sido definida por Tulving (1972) como el almacenamiento a largo plazo de eventos experimentados por la persona, tratándose de información autobiográfica; 
la MT, según Baddeley \& Hitch (1974), es un sistema que consta de tres componentes: un ejecutivo central (encargado de la selección y planificación, nexo entre los otros dos subsistemas y la memoria de largo plazo) y dos subsistemas que son el bucle fonológico (que sostiene información verbal) y la agenda visoespacial (que sostiene información visual y espacial). Sin embargo, Baddeley (2000) encontró que hay una serie de fenómenos que no son capturados fácilmente por el modelo original, proponiendo un cuarto componente que denominó buffer episódico. Éste se compone de un sistema de capacidad limitada que proporciona el almacenamiento temporal de la información contenida en un código multimodal, que es capaz de unir información de los sistemas auxiliares, y desde la memoria a largo plazo, en una representación episódica unitaria, es un sistema que está estrechamente relacionado con la funcionalidad ejecutiva, en tanto posibilita el establecimiento de representaciones para acciones futuras.

Por su parte, la memoria fuente (MF), del inglés Source Memory, se ha considerado un tipo de ME (Rosselli, Matute y Ardila, 2010) y ha sido considerada por Cycowicz, Friedman, Snodgrass \& Duff (2001) un tipo de ME en estrecha relación con el funcionamiento frontal y ontogenéticamente de aparición tardía. La MF es descrita por Johnson, Hashtoudi \& Lindsay (1993) y Wheeler, Stuss \& Tulving (1997), quienes diferencian entre tres tipos de MF. La MF interna, que hace alusión a juzgar si uno realmente realizó o imaginó hacer una acción; la $M F$ externa que se refiere a juzgar quién realizó una acción, y finalmente la $M F$ interna-externa o yo-otro, que es la capacidad de juzgar si es uno mismo u otra persona quien realiza una acción.

Por otro lado, la MP es conceptualizada por Brandimonte, Einsteinand \& McDaniel (1996) como el conjunto de habilidades que se utilizan para recordar en el momento de realizar una acción prevista, o pensamiento, en algún momento futuro. En algunas ocasiones, la acción debe ser realizada en un momento determinado (MP basada en el tiempo), mientras que en otros sólo se puede realizar la acción cuando se produce un evento en particular (MP basada en eventos). Se acepta comúnmente que las tareas de MP implican dos componentes: un componente retrospectivo y un componente prospectivo.

De acuerdo con las características de TEA y la importancia de la memoria no solo para los procesos de aprendizaje, sino para el desarrollo humano integral, es relevante realizar un artículo de revisión en el cual se evidencie el estado actual de los sistemas de memoria (ME, MT y MP) en niños con TEA sin déficit intelectual, describiendo las dificultades que presentan en el funcionamiento de los sistemas de memoria, lo que facilitará evidenciar las potencialidades sobre las cuales los maestros deben intervenir para propiciar procesos de enseñanzaaprendizaje. Es así, que desde un punto de vista pedagógico, conocer el funcionamiento de la memoria le posibilitará al docente crear una línea base que permita generar diferentes estrategias pedagógicas que vayan en pro del mejoramiento de los aspectos que se encuentran deteriorados, pero sobretodo, potencializar aquellos que están intactos a partir de los cuales 
las estrategias pedagógicas utilizadas tendrán efectos, no solo en el contexto escolar, sino en el desarrollo humano integral, asumiendo que la utilización de las explicaciones neurocientíficas al servicio del contexto escolar es una necesidad imperante en la actualidad (De la Peña, 2005; D’Amato, Crepeau-Hobson, Huang \& Geil, 2005; Goswami, 2006; Solovieva, 2014; Margulis, 2009; Bordignon, Endres, Trentini y Bosa, 2015).

\section{Metodología}

Se realizó una búsqueda de artículos resultados de investigación en diferentes bases de datos, entre las que se destacan EBSCO, Eric, APA PsicNET, PubMed, entre otras. Se utilizaron palabras clave como: autism, autism spectrum disorder, children, memory, episodic memory, working memory, source memory, semantic memory.

Los criterios de inclusión fueron:

Año de publicación: las investigaciones fueron aquellas cuyo periodo estaba comprendido desde 2009 hasta 2015.

Edad de la muestra: las investigaciones rastreadas utilizan muestras de diferentes edades (3, 5-8, 6-16, 8-16 años) y los diversos hallazgos muestran que los compromisos de los diferentes sistemas de memoria no varían dependiendo de la edad. Por lo tanto, la edad no fue una variable demográfica de inclusión, pero se consideraron de importancia para la actual revisión, los antecedentes investigativos cuya muestra hayan sido niños y adolescentes.

Clasificación del autismo: se encontraron investigaciones que abordaban otros trastornos del espectro autista diferentes al TEA sin déficit intelectual acompañante, pero sólo se retomaron estas últimas investigaciones, dado que la pretensión es caracterizar el funcionamiento de los diferentes sistemas de memoria en elTEA sin déficit intelectual acompañante, pues se requiere precisar que dicho funcionamiento no se vea explicado por otra condición que haga parte de los trastornos del neurodesarrollo (DSM-5, 2014).

\section{Resultados}

Pérez y Martínez (2014) realizaron una revisión teórica de la evidencia empírica sobre el perfil cognitivo del AAF y el Síndrome de Asperger (SA), y advirtieron que autores como Boucher (1981), Boucher \& Warrington (1976), Bowler, Gardiner \& Grice (2000) han encontrado que las personas con AAF presentan desempeños satisfactorios frente a tareas que requieren modalidades sensoriales visuales, lo que implica afirmar que codifican, organizan, almacenan y evocan información visual tal y como lo realizaría un grupo de personas sin trastornos del neurodesarrollo, mientras que presentan compromisos importantes en aquellas tareas que 
requieren de una memoria declarativa, específicamente aquella información episódica (Bowler et al., 2000, quienes encontraron este déficit en personas con SA) y de carácter verbal, hallazgo que no es consistente con el encontrado por Blair, Frith, Smith, Abell \& Cipolotti (2002), quienes identificaron un desempeño normal en tareas de memoria verbal, especialmente las relacionadas con estímulos auditivos.

\section{MT en personas con AAF o TEA sin déficit intelectual}

Con relación a la MT se han encontrado diferentes resultados. Schuh et al. (2012) reportaron deficiencias globales en la MT, sugiriendo que las personas con AAF tienen dificultades en el proceso de almacenamiento de la información, tanto en las tareas simples como complejas, y en las tareas de atención dividida (Yerys, Wallace, Jankowski, Bollich \& Kenworthy, 2011), llevando a un grupo de personas con este diagnóstico a presentar dificultades en la realización de tareas de la vida cotidiana, lo cual es inconsistente con los hallazgos de Andersen et al. (2013) y Jiang et al. (2014) quienes encontraron deficiencias específicas en la MT verbal en personas con AAF y problemas atencionales y alteraciones en la MT espacial (Jiang et al., 2014; Bordignon et al., 2015). Lo sugerido por Andersen et al. (2013) lleva a inferir que aquellas personas con AAF sin problemas atencionales, tienen intacta la MT verbal.

Williams, Goldstein, Carpenter \& Minshew (2005), encontraron que esta población, en comparación con un grupo sin TEA, tenía ejecuciones similares en tareas de MT en las que se requería del bucle articulatorio y presentaba un rendimiento más pobre en aquellas tareas que implicaban la agenda visoespacial. Los autores terminan afirmando que las personas con autismo tienen intacto lo relacionado al campo verbal y presentan dificultades en la agenda viso-espacial, lo cual es consistente con Cui, Gao, Chen, Zou \& Wang (2010), quienes encontraron que las personas con TEA, específicamente personas con SA, tienen ventaja en el almacenamiento del bucle fonológico, pero desventaja en el almacenamiento visuespacial. Tales hallazgos son discutidos por So, Lui, Wong \& Sit (2015), quienes encontraron que las personas con TEA tienen fuertes habilidades en el aspecto de razonamiento espacial abstracto debido a los resultados obtenidos en la prueba de matrices progresivas de Raven, la cual al basarse en estrategias visuales para realizar tareas referentes a gestos de identificación, tuvo una correlación positiva, sugiriendo fortalezas en el aspecto viso-espacial. Este hallazgo confirma resultados de estudios anteriores, específicamente el de Soulieres, Zeffiro, Girard \& Mottron (2011), quienes afirman que las personas con TEA no están afectados en tareas que requieran MT visoespacial.

A pesar de las deficiencias encontradas en la MT en personas con TEA, existen estudios que han ahondado en procedimientos para su intervención (Alloway et al., 2010). Baltruschat et al. (2012), argumentan que la MT puede ser susceptible de modificación en algunas personas con AAF a través de procedimientos de intervención dirigidos a comportamientos básicos, 
como el refuerzo positivo. Con relación a esto, Phelan, Filliter \& Johnson (2011) indican que el rendimiento de la memoria verbal en las personas con TEA está relativamente intacto, pero puede mejorar su desempeño a través de estímulos externos. Tales hallazgos apoyan el supuesto del Task Support Hypothesis (TSH; Bowler, Matthews \& Gardiner, 1997), en la cual se afirma que la presencia de señales externas facilita el desempeño de las personas con TEA en las pruebas de memoria.

\section{ME en personas con AAF oTEA sin déficit intelectual}

En cuanto a la ME, diferentes autores han encontrado resultados consistentes. Por su parte Wheeler et al. (1997) afirman que la ME está a un nivel de conciencia autonoética, pues ésta requiere de la evocación de una experiencia propia, siendo de esta manera un recuerdo subjetivo.

Andreano \& Cahill (2009) han reportado que un área que ha recibido recientemente una atención considerable en la investigación del autismo, y en la que se ha informado de diferencias de género en la población normal, es la memoria autobiográfica. Con relación a ésta, Goddard et al.(2014), exploraron las diferencias de género en ella y encontraron que un déficit en la recuperación de esta memoria fue más característico de los participantes de género masculino con TEA, indicando que las mujeres tanto del grupo TEA como control, tuvieron un mejor desempeño quienes mostraron una capacidad de generar recuerdos más detallados y emocionales que los hombres y adquisición de estrategias compensatorias superiores debido a un mayor procesamiento autorreferencial (Lai, Lombardo, Pasco, Ruigrok, Wheelwright, Sadek et al., 2011). En este sentido, Goddard et al. (2014) sostienen que la memoria autobiográfica de las personas con TEA se caracteriza por una dificultad en la recuperación de recuerdos específicos de eventos contextualizados en tiempo y lugar, sosteniendo que los déficits de ésta se explican por las alteraciones cognitivas en la conciencia autonoética, reflejándose allí las dificultades que tienen las personas con TEA en lo relacionado con el sistema de ME.

Por otra parte, Terret et al. (2013), Zalla et al. (2013) y Bordignon et al. (2015) afirman que existen compromisos de ME en personas con AAF, específicamente con el pensamiento futuro episódico, encontrando dificultades importantes para la evocación de hechos pasados (es decir, implicación en la conciencia autonoética), así como para imaginar nuevos eventos. Particularmente, Trontel et al. (2013) y Southwick et al.(2011) han encontrado déficits en ME utilizando el test de memoria y aprendizaje (del inglés Test of Memory and Learning -TOMAL, 1994), lo que los lleva a afirmar que las personas con diagnóstico de TEA presentan peores ejecuciones que el grupo control en las subpruebas de recuerdo de objetos, recuerdo selectivo visual y memoria de caras, hallazgo consistente con los de Wilkinson, Best, Minshew \& Strauss (2010), quienes encontraron que personas con AAF tienen deficiencias en la conciencia de reconocimiento de caras. Por su parte, Southwick et al. (2013) afirman que los pacientes 
Caracterización de la memoria en niños con trastorno del espectro autista sin déficit intelectual...

con TEA tienen ejecuciones esperadas en aquellos subtests del TOMAL que posibilitan la evaluación de la memoria compuesta, verbal y demorada, teniendo ejecuciones más pobres en los subtests de memoria de ubicación, imitación manual y recuerdo demorado selectivo de palabras, lo que pudiera sugerir compromisos en la memoria no verbal.

Varios estudios han encontrado déficits en ME al relacionarla con el rendimiento de la MF tanto a nivel interno (Cornett et al., 2013; Bigham et al., 2010) como a nivel interna-externa o yo-otro (Lelii et al., 2012 y Lind et al., 2009).

Cornett et al. (2013) se han interesado por investigar la relación existente entre la memoria de reconocimiento en personas con autismo y los acontecimientos vividos y presenciados y han encontrado que las personas con autismo recuerdan los acontecimientos que les sucedieron con menos precisión en comparación con el grupo control, reflejando la dificultad que tienen para almacenar eventos auto-experimentados. Por su parte, Bigham et al. (2010) diferencian entre familiaridad y recuerdo, definiendo la familiaridad como una sensación consciente de que se ha experimentado un estímulo sin recordar necesariamente otra información, mientras que el recuerdo es el estímulo de señales reconocidas en información contextual con experiencia en el episodio en el que se encontró dicho estímulo. Tales autores encontraron que la familiaridad se encuentra intacta en personas con TEA, concretamente en aquellas con AAF, mientras que el recuerdo está alterado, evidenciando los déficits en ME que presenta esta población.

Por su parte, Lelii et al. (2012) se preocuparon por examinar el autoreconocimiento en personas con TEA mediante el instrumento denominado Self-Other Action Memory y encontraron que esta población tiene peores rendimientos en tareas que requieran autorreconocimiento y concluyen que las personas con TEA presentan dificultades significativas en memoria internaexterna, hallazgo consistente con el de Lind et al. (2009) quienes aseveran que en el autismo se tienen dificultades para unir diversos episodios, reflejándose allí las alteraciones encontradas en la ME.

En síntesis, la ME se encuentra significativamente alterada en AAF (Terrett et al., 2013) y en TEA (Lind et al., 2009; Southwick et al., 2011; Zalla et al., 2011), así como también la MF (Lind et al., 2009 y Lelii et al., 2012), como uno de los subtipos de la ME.

\section{MT - ME y su relación con la MP en personas con TEA}

Diferentes estudios, especialmente el de Brenner et al. (2014), han encontrado que la MT opera en conjunto con la MP, razón que los lleva a afirmar que las personas con TEA que presentan alteraciones en la MT tienen implicaciones significativas en el rendimiento de la MP, máxime cuando son personas de corta edad, lo que implica que el procesamiento temporal en el TEA mejora conforme se avanza el ciclo vital. 
Por otra parte, Maister et al. (2011) y Gil et al. (2012) encuentran que la MP está en estrecha relación con el desempeño de la ME en personas con TEA. Afirman que los recuerdos episódicos se caracterizan por su especificidad en un contexto espacio-temporal determinado. Así, mientras que el tratamiento de la información temporal es crucial para el desarrollo de la $\mathrm{ME}$, la relación es bidireccional en el sentido de que la ME es fundamental para hacer juicios sobre la información temporal.

Los resultados sobre la MP basada tanto en el tiempo como en eventos en las personas con diagnóstico de TEA, han sido poco precisos. Por una parte, con relación a la MP basada en el tiempo, algunas investigaciones (Altgassen et al., 2009; Maister et al., 2011 y Williams, Boucher, Lind \& Jarrold, 2013) han afirmado déficits, mientras que otros hallazgos han encontrado intacta esta memoria (Gil et al., 2012). Por otra parte, en cuanto a la MP basada en eventos, algunos estudios (Jones et al., 2011; Brandimonte, Filippello, Coluccia, Altgassen \& Kliegel, 2011 y Yi et al., 2014) aseguran que se encuentra deteriorada, mientras que otros (Altgassen, Schmitz-Hu“bsch \& Kliegel, 2010 y Williams et al., 2013) afirman que no presenta compromisos. Dichas discrepancias pueden verse explicadas por el tipo de técnicas de recolección de datos utilizadas en los diferentes estudios, así como por los tamaños muestrales. Por otra parte, con relación a la MP basada en eventos, Jones et al. (2011), Brandimonte et al. (2011) y Yi et al. (2014), al evaluar una muestra de adolescentes con TEA y compararlos con un grupo control, y utilizando como instrumento de medición el Test de Memoria Conductual de Rivermead, concluyeron que la población con TEA tiene un funcionamiento esperado en lo relacionado con la MP, mientras que Altgassen et al. (2010) y Williams et al. (2013) encontraron funcionamientos anómalos en la misma (MP eventos) al evaluar a través de tareas de memoria prospectiva, la MP de eventos en una muestra de niños con TEA y comparar las ejecuciones con un grupo control. Es probable que dichas discrepancias se relacionen con la edad de las muestras y el tipo de técnica de recolección utilizada, por lo que no puede ser un asunto concluyente el que la MP de eventos esté intacta o deteriorada en las personas con TEA; sin embargo, desde allí se puede sugerir cuidadosamente que en la población con TEA tiende a existir peores ejecuciones en la MP de eventos mientras más pequeños sean.

Otros estudios relacionados con la MP basada en eventos fueron los desarrollados por Maister et al. (2011) y Gil et al. (2012) cuyos resultados son inconsistentes. Por su parte, Maister et al. (2011), al evaluar el procesamiento temporal en personas con y sin TEA, encontraron que el grupo con TEA tuvo un desempeño significativamente más pobre en la reproducción de corta (relacionado con la memoria a corto plazo) y larga duración (relacionado con la MP) del procesamiento temporal en comparación con el grupo control, lo que los lleva a estimar que la MP de eventos está deteriorada en las personas con TEA. Mientras que Gil et al. (2012), encontraron que las personas con TEA realizaron correctamente las tareas de los dos intervalos de tiempo (largo y corto), proporcionando evidencia que indica que la percepción del tiempo en las personas con autismo podría estar intacta. 
Jones et al. (2011) se preocuparon por identificar las habilidades cotidianas de memoria de las personas con TEA a través del Test de Memoria Conductual de Rivermead y encontraron dificultades en esta memoria en las personas con TEA, incluyendo compromisos en la MP basada en eventos. Adicionalmente, se interesaron por identificar la existencia de una probable relación entre las puntuaciones obtenidas de la medición de MP de eventos con las habilidades sociales y comunicativas y encontraron una correlación positiva, lo que los lleva a afirmar que una persona con TEA presentará compromisos en MP dadas las deficiencias persistentes en la comunicación e interacción social.

En el estudio de Yi et al. (2014) se investigó la MP basada en eventos y sus correlatos cognitivos en personas con TEA, encontrando que las personas con TEA tenían puntuaciones significativamente más bajas en la tarea MP que el grupo control. Hallazgo contrario a lo encontrado por Williams et al. (2013), quienes al estudiar la MP basada en eventos y tiempo, afirman que la MP basada en eventos está intacta en personas con TEA, mientras que la MP basada en el tiempo se encuentra disminuida.

Los hallazgos previamente descritos con relación a la MP de eventos, muestran resultados que no permiten aseverar si el funcionamiento de la MP basada en eventos está intacta o deteriorada en la población con TEA, lo que pudiera esclarecerse a partir de investigaciones con muestras representativas y que conformen diferentes grupos dependiendo de la edad cronológica.

\section{Procesos de memoria en el TEA sin déficit intelectual}

Con relación a los procesos de la memoria, diferentes investigaciones (Southwick et al., 2011 y Wojcik et al., 2013) han encontrado que la codificación está alterada en personas con TEA. Wojcik et al. (2013) utilizaron el Paradigma de Sensación de Saber (FOK- del inglés Feeling-ofKnowing), el cual es similar a lo que experimentan las personas cuando dicen que tienen cierta información en la "punta de la lengua”, es decir, ese momento en el que no pueden recuperar la palabra, pero 'saben' que van a ser capaces de recuperarla después. Estos autores distinguen entre dos tareas de dicho paradigma: episódica y semántica. La tarea FOK episódica implica un nuevo aprendizaje y por lo tanto requiere la codificación de nueva información, mientras que la tarea FOK semántica se basa en el conocimiento de hechos y por lo tanto no evalúa codificación. Encontraron que personas con TEA tuvieron un rendimiento disminuido en la tarea FOK episódica, lo cual, según los autores, podría estar relacionado con la dificultad para codificar la información. Así mismo, Southwick et al. (2011) hallaron que la codificación y organización de la información son los principales factores que limitan el rendimiento de memoria en el autismo.

Por otra parte, diferentes estudios, especialmente el de Brezis et al., (2014) han encontrado que la recuperación está alterada en personas con TEA, al afirmar que tienen dificultad para 
recordar información compleja sobre los estímulos simples y aislados, hallazgo ante el cual Mattison, Dando \& Ormerod (2014) plantearon que por medio de la técnica de "Sketch reinstatement of context" (Sketch-RC), que consiste en dibujar situaciones experimentadas de una manera abstracta, se posibilita que las personas con TEA evoquen información de un evento episódico, generando para ello representaciones perceptuales en lugar de procesos verbales para acceder a recuerdos episódicos.

Los resultados anteriores están en correspondencia con lo hallado por Erdodi et al. (2013) y McGonigle-Chalmers \& McSweeney (2013), quienes encontraron que personas con TEA tienen mejor capacidad visual que verbal, la cual varía de acuerdo con la complejidad de la información, teniendo dificultades con la velocidad de procesamiento de tareas complejas. Con relación a esto último, Tsatsanis et al. (2011) examinaron en qué medida la organización y el estilo de procesamiento impacta en el análisis perceptual y recuerdo de la información visual compleja. Identificaron que el grupo TEA mostró un estilo de procesamiento "partes a partes", postulando que los sujetos con autismo desafían el factor de la complejidad mediante este tipo de estrategia que consiste en percibir sólo partes aisladas y no en contexto global, lo que resulta en una imagen fragmentada, por lo que es probable que procesen la información compleja analizándola en sus partes-componentes y no por "partes al todo", lo cual alude a la capacidad de percibir la totalidad del contexto. Congruente con lo anterior, es lo reportado por Mammarellaa, Giofre, Caviola, Cornoldi \& Hamilton (2014) quienes aseguran que las personas con TEA tienen un estilo de procesamiento detalle-centrado, postulando que tienen la facilidad de segmentar en partes, pero por el contrario, luchan cuando tienen que tratar con una organización global del material. Estas debilidades en algunos aspectos y las fortalezas en otros, es lo que Frith (1989) describe como la teoría de la coherencia central débil.

Finalmente, diferentes estudios han encontrado que la función de la planificación, aspecto que se encuentra bajo el dominio de la función ejecutiva, tiene un impacto significativo en la MT (Salcedo, Moreno, Ruiz \& Ferrin, 2013) y MP (Rajendran et al., 2011). Salcedo et al. (2013) por su parte, plantean que aunque la planificación es un proceso independiente de los otros dominios cognitivos, funciona como un moderador de rendimiento en la MT, mientras que Rajendran et al. (2011) afirman que cuando la MP se encuentra alterada en elTEA, es de esperarse que también lo estén los procesos de planificación e inhibición propios de la función ejecutiva. 


\section{Discusión}

Los sistemas de memoria afectados en las personas con TEA sin déficit intelectual son la MT, ME, MP y algunos procesos de memoria como la codificación, organización y la recuperación de información, así como la planificación e inhibición propias de la función ejecutiva. Con relación a la MT, se han encontrado diferentes resultados; mientras algunas investigaciones, en especial la de Schuh et al. (2012), reportaron deficiencias globales en la MT, otros estudios (Andersen et al., 2013 y Jiang et al., 2014) encontraron deficiencias específicas en la MT verbal y alteraciones en la MT espacial. Por otra parte, los resultados sobre la ME son consistentes debido a que la generalidad de estudios abordados reportan dificultades en las personas con TEA en dicha memoria. En el estudio de Goddard (2014) lo hace al explorar las diferencias de género en la memoria autobiográfica, los hallazgos de Terret et al. (2013) y Zalla et al. (2013) lo evidencian al encontrar que las personas con TEA tienen dificultades en el pensamiento futuro episódico y Cornett et al. (2013), Bigham et al. (2010), Lelii et al. (2012) y Lind et al. (2009) reportan tal déficit al relacionar la ME con la MF. Adicionalmente, se ha encontrado que la MT opera en conjunto con la MP (Brenner et al., 2014) y está en estrecha relación con el desempeño de la ME en personas con TEA (Maister et al., 2011; Gil et al., 2012).

Los resultados sobre la MP basada en el tiempo en personas con TEA han sido poco precisos; algunos hallazgos (Altgassen et al., 2009 y Maister et al., 2011) sugieren déficit en MP, mientras que otros estudios (Gil et al., 2012) han encontrado intacta esta memoria.

Finalmente, con relación a los procesos de la memoria, diferentes investigaciones han encontrado que la codificación (Southwick et al., 2011 y Wojcik et al., 2013) y la recuperación (Brezis et al., 2014) está alterada en personas con TEA. Así mismo, Erdodi et al. (2013) afirman que las personas con TEA tienen mejor capacidad visual que verbal, la cual varía de acuerdo con la complejidad de la información.

\section{Conclusiones}

Los niños con TEA presentan claras deficiencias en la ME, pues se les dificulta evocar las acciones realizadas y el contexto de las mismas, así como manipular información actual para generar acciones y pensamientos futuros, lo que indica escasas estrategias para actuar de manera ejecutiva frente a las demandas actuales y prospectivas. Ello se ve explicado por los déficits en la MT, en la cual presentan compromisos en los diferentes componentes, buffer fonológico, agenda visoespacial y buffer episódico, en tanto este es un modulador mental que posibilita la generación de estrategias, no solo para evocar información, sino para utilizar la información según unas metas y propósitos determinados. Del mismo modo, aun teniendo 
intacta la memoria semántica, es de esperarse que tenga inhabilidad para utilizar la información semántica y actuar coherente con ello, tanto para la solución de situaciones presentes como futuras, dados los compromisos en la MT. Finalmente, se precisa que los niños con TEA sin déficit intelectual, presentan dificultades relacionadas con la codificación y evocación de información verbal y espacial, lo que indica que los estímulos a presentarse para la adecuada codificación, organización y almacenamiento deben tener carácter no verbal y visual.

Reconocer el funcionamiento de la memoria en niños con TEA sin déficit intelectual para los profesionales que trabajan con la primera infancia resulta una herramienta fundamental para la creación de estrategias pedagógicas coherentes con la manera que tiene de aprender el cerebro, específicamente de la población con este diagnóstico y, en esta medida, será posible la aproximación a los procesos de enseñanza aprendizaje. Es de destacar que un educador genera y crea las alternativas básicas necesarias y suficientes para que los niños de la primera infancia, adquieran con las mínimas dificultades posibles, los procesos de lectura, escritura y cálculo en el momento propicio, y ello se logra no solo reconociendo que el cerebro tiene una manera específica y particular de 'aprender', sino reconociendo que la dinámica que allí suceda se debe a la relación bidireccional y transaccional de las características propias de ese cerebro, con los contextos escolar, familiar y social. Se asume desde una multifactorialidad la importancia de los contextos de desarrollo (aspectos cognitivos, afectivo-sociales, escolares y familiares) y ello resulta ser una responsabilidad asumida desde el perfil del educador, es por esto, que no solo se reconoce la manera de aprender desde los recursos cerebrales, sino cómo las influencias contextuales posibilitan en interacción con esta individualidad, el desarrollo humano integral.

Sería interesante que investigaciones futuras delimiten y realicen comparaciones entre diferentes rangos de edad y estimar el funcionamiento de los diferentes sistemas de memoria en niños con TEA sin y con déficit intelectual y grupo control. 


\section{Referencias}

Asociación Americana de Psiquiatría (2014). Manual diagnóstico y estadístico de los trastornos mentales (DSM-5), 5 Ed. Arlington, VA, Asociación Americana de Psiquiatría.

American Psychiatric Association. (2002). Manual diagnóstico y estadístico de los trastornos mentales IVTR. Barcelona, España: APA.

Alloway, T.P. \& Alloway, R.G. (2010). Investigating the predictive roles of working memory and IQ in academic attainment. Journal of Experimental Child Psychology, 80, 606-621

Altgassen, M., Williams, T., Bölte, S. \& Kliegel, M. (2009)Time-Based Prospective Memory in Children with Autism Spectrum Disorder. Brain impairment, 10(1), 52-58

Altgassen, M., Schmitz-Hu“bsch, M. \& Kliegel, M. (2010). Event-based prospective memory performance in autism spectrum disorder. Journal of Neurodevelopmental Disorders, 2, 2-8 http: / / dx.doi.org/10.1007/s11689-009-9030-y

Andersen, Hovik, Skogli, Egeland \& Øie (2013). Symptoms of ADHD in Children with HighFunctioning Autism Are Related to Impaired Verbal Working Memory and Verbal Delayed Recall. PLoS ONE 8(5): e64842. doi:10.1371/journal.pone.0064842

Andreano, J.M. \& Cahill, L. (2009). Sex influences on the neurobiology of learning and memory. Learning and Memory, 16(4), 248-266.

Baddeley, A. (1999). Memoria humana, teoría y práctica. Aravaca, España: McGraw-Hill

Baddeley, A. \& Hitch, G. (1974). Working memory. In G.H. Bower, (Ed.), The psychology of learning and motivation:Advances in research and theory (pp. 47-90). New York, United States: Academic Press.

Baddeley, A.D. (2000). The episodic buffer: A new component of working memory? Trends in Cognitive Sciences, 4, (11), 417-423.

Baltruschat, L. et al. (2012). The effects of multiple exemplar training on a working memory task involving sequential responding in children with autism. The Psychological Record, 62, 549-562

Bigham, S., Boucher, J., Mayes, A. \& Anns, S. (2010) Assessing Recollection and Familiarity in Autistic Spectrum Disorders: Methods and Findings. JAutism DevDisord, 40, 878-889. doi: 10.1007/s10803-010-0937-7

Bordignon, S., Endres, R., Trentini, C. \& Bosa, C. (2015). Memory in Children and Adolescents with Autism Spectrum Disorder: A Systematic Literature Review. Psychology, \& Neuroscience, 8(2), 211-245. 
Bowler, D.M., Matthews, N.J. \& Gardiner, J.M. (1997). Asperger's syndrome and memory: Similarity to autism but not amnesia. Neuropsychologia, 35, 65-70.

Boucher, J., Mayes, A. \& Bigham, S. (2006). Memory in autistic spectrum disorder. Psychol Bull, 138(3), 458-96. doi: 10.1037/a0026869.

Boucher, J. (1981). Immediate free recall in early childhood autism: Another point of behavioural similarity with the amnesic syndrome. British Journal of Psychology, 72, 211-215.

Boucher, J. \& Warrington, E.K. (1976). Memory deficits in early infantile autism: some similarities to the amnesic syndrome. British Journal of Psychology, 67(1), 73-87.

Bowler, D.M., Gardiner, J.M. \& Grice, S.J. (2000). Episodic memory and remembering in adults with Asperger's syndrome. Journal of Autism and Developmental Disorders, 30(4), 295-304.

Blair, R.J.R., Frith, U., Smith, N., Abell, F. \& Cipolotti, L. (2002). Fractionation of visual memory: agency detection and its impairment in autism. Neuropsychologia, 40, 108-118.

Brandimonte, M.A., Einstein, G.O. \& McDaniel, M.A. (1996). Prospective memory: Theory and applications. Mahwah, NJ: Lawrence Erlbaum.

Brandimonte, M.A., Filippello, P., Coluccia, E., Altgassen, M. \& Kliegel, M. (2011). To do or not to do? Prospective memory versus response inhibition in autism spectrum disorder and attention-deficit/hyperactivity disorder. Memory, 19, 56-66 http://dx.doi.org/10.1 080/09658211.2010.535657

Brenner, L., Shih, H., Colich, N., Sugar, C., Bearden, C. \& Dapretto, M. (2014). Time Reproduction Performance Is Associated with Age and Working Memory in High-Functioning Youth with Autism Spectrum Disorder. Autism Research, 8, 29-37. doi: 10.1002/aur.1401.

Brezis, R., Galili, T., Wong, T. \& Piggot, J. (2014). Impaired Social Processing in Autism and its Reflections in Memory: A Deeper View of Encoding and Retrieval Processes. Journal Autism Development Disorder, 44, 1183-1192. doi: 10.1007/s10803-013-1980-y

Cycowicz,Y., Friedman, D., Snodgrass, J. \& Duff, M. (2001). Recognition and source memory for pictures in children and adults. Neuropsychology, 39, 255-267.

Cornett, K., Miora, D., Fass, T. \& Dixon, D. (2013). Memory Functioning for Personally Experienced and Witnessed Events in Children with Autism and the Implications for Educators, Mental Health Professionals, and the Law. Journal of Applied Research on Children: Informing Policy for Children at Risk, 4(2), 1-12

Cui, J., Gao, D., Chen, Y., Zou, X. \& Wang,Y. (2010). Working Memory in Early- School Age Children with Asperger's Syndrome. Journal Autism DevelopmentDisord, 40, 958-967 doi: 10.1007/s10803-010-0943-9 
Caracterización de la memoria en niños con trastorno del espectro autista sin déficit intelectual...

D’Amato, R.C., Crepeau-Hobson, F., Huang, L.V. \& Geil, M. (2005). Ecological neuropsychology: an alternative to the deficit model for conceptualizing and serving students with learning disabilities. Neuropsychological Review, 15(2), 97-103.

De la Peña Álvarez, M.C. (2005). Neuropsicopedagogía. La psicopedagogía del futuro. Tribuna Libre, (2). Recuperado de http:/ / comunidad- escolar.pntic.mec.es/762/tribuna2.html

Erdodi, L., Lajiness-O’Neill, R. \& Shmitt, T. (2013). Learning Curve Analyses in Neurodevelopmental Disorders: Are Children with Autism Spectrum Disorder Truly Visual Learners?J Autism DevDisord, 43, 880-890. doi: 10.1007/s10803-012-1630-9

Frith, U. (1989). Autism: Explaining the enigma. Oxford, UK: Basil Blackwell.

Gil, S., Chambres, P., Hyvert, C., Fanget, M. \& Droit-Volet, S. (2012). Children with Autism Spectrum Disorders Have "The Working Raw Material” for Time Perception. PLoS ONE, 7(11), e49116. doi: 10.1371/journal.pone.0049116

Goddard, L., Dritschel, B. \& Howlin, P. (2014). A Preliminary Study of Gender Differences in Autobiographical Memory in Children with an Autism Spectrum Disorder.J Autism DevDisord, 44, 2087-2095. doi: 10.1007/s10803-014-2109-7

Goswami, U. (May, 2006). Neuroscience and education: from research to practice? Nature Reviews Neuroscience, 7, 406-413. doi: 10.1038/nrn1907

Johnson, M.K., Hashtoudi, S. \& Lindsay, D.S. (1993). Source monitoring. Psychological Bulletin, 114, 3-28. doi: 10.1037/ 0033-2909.114.1.3.

Jiang, Y., Capistrano, C. \& Palm, B. (2014). Spatial Working Memory in Children with HighFunctioning Autism: Intact Configural Processing But Impaired Capacity. Journal of Abnormal Psychology, 123 (1), 248-257.doi: 10.1037/a0035420

Jones, C., Happe, F., Pickles A., Marsden, A., Tregay, J., Baird G., Simonoff, E. \& Charman, T. (2011). Everyday Memory' Impairments in Autism Spectrum Disorders. Journal Autism DevelopmentDisord, 41, 455-464 doi:10.1007/s10803-010-1067-y.

Lai, M.C., Lombardo, M.V., Pasco, G., Ruigrok, A.N., Wheelwright, S.J., Sadek, S.A. et al. (2011). A behavioral comparison of male and female adults with high functioning autism spectrum conditions. PLoS ONE, 6(6), e20835.

Lelii, S. \& Wellman, H. (2012). Delayed Self Recognition in Autism: A Unique Difficulty? Res Autism SpectrDisord, 6(1), 212-223. doi: 10.1016/j.rasd.2011.05.002

Lind, S., \& Bowler, D. (2009). Recognition Memory, Self-Other Source Memory, and Theory of Mind in Children with Autism Spectrum Disorder. J Autism DevDisord, 39, 1231-1239. doi 10.1007/s10803-009-0735-2 
Maister, L. \& Plaisted-Grant, K. (2011). Time perception and its relationship to memory in Autism Spectrum Conditions. Developmental Science, 1311-1322. doi: 10.1111/j.14677687.2011.01077.x

Mammarellaa, I., Giofre, D., Caviola, S., Cornoldi, C. \& Hamilton, C. (2014). Visuospatial working memory in children with autism: The effect of a semantic global organization. Research in DevelopmentalDisabilities, 35, 1349-1356. doi: 10.1016/j.ridd.2014.03.030

Margulis, L. (2009). Funcionamiento de los sistemas de memoria en niños con Trastorno Autista y Trastorno de Asperger. Revista Argentina de Neuropsicología, 13, 29-48.

Mattison, M., Dando, C. \& Ormerod, T. (2014). Sketching to Remember: Episodic Free Recall Task Support for Child Witnesses and Victims with Autism Spectrum Disorder. J Autism DevDisord, 45(6),1751-65. doi: 10.1007/s10803-014-2335-z

McGonigle-Chalmers. \& McSweeney, M. (2013). The Role of Timing in Testing Nonverbal IQ in Children with ASD. Journal of Autism, \& Developmental Disorders, 43(1), 80-90. doi:10.1007/s10803-012-1545-5

Pérez, P. y Martínez, L. (2014). Perfiles cognitivos en el Trastorno Autista de Alto Funcionamiento y el Síndrome de Asperger. CES Psicología, 7(1), 141-155.

Phelan, H., Filliter, J. \& Johnson, S. (2011). Brief Report: Memory Performance on the California Verbal Learning Test - Children's Version in Autism Spectrum Disorder. J Autism DevDisord, 41, 518-523. doi:10.1007/s10803-010-1069- 9

Rajendran, G., Law, A., Logie, R., Meulen, M., Fraser, D. \& Corley, M. (2011). Investigating Multitasking in High-Functioning Adolescents with Autism Spectrum Disorders Using the Virtual Errands Task. J Autism DevDisord, 41, 1445-1454. doi: 10.1007/s10803-010-1151-3

Rosselli, M., Matute, E. y Ardila, A. (2010). Neuropsicología del desarrollo infantil. México D.F. México: Manual Moderno.

Salcedo, M., Moreno, J., Ruiz, M. \& Ferrin, M. (2013). Evaluation of Planning Dysfunction in Attention Deficit Hyperactivity Disorder and Autistic Spectrum Disorders Using the Zoo Map Task. Child Psychiatry Hum Dev, 44, 166-185. doi: 10.1007/s10578-012-0317-y

Schuh \& Eigsti. (2012). Working Memory, Language Skills, and Autism Symptomatology. Behavioral Sciences, 2, 207-218.doi:10.3390/bs2040207

So, W., Lui, M., Wong, T. \& Sit, L. (2015). The Use of Hand Gestures to Communicate About Nonpresent Objects in Mind among Children with Autism Spectrum Disorder. Journal of Speech, Language, and Hearing Research, 58, 373-382.doi: 10.1044/2015_JSLHR-L-14-0213

Solovieva, Y. (2014). Intervención neuropsicológica infantil: diversidad de posibilidades. Revista Chilena de Neuropsicología, 9(2), 46-48. 
Caracterización de la memoria en niños con trastorno del espectro autista sin déficit intelectual...

Soulieres, I., Zeffiro, T.A., Girard, M.L. \& Mottron, L. (2011). Enhanced mental image mapping in autism. Neuropsychologia, 49, 848-857.

Southwick, J.S., Bigler, E.D., Froehlich, A., DuBray, M.B., Alexander, A.L., Lange, N. \& Lainhart, J.E. (2011). Memory Functioning in Children and Adolescents with Autism. Neuropsychology, 25(6), 702-710

Terrett, G., Rendell, P., Saunders, S., Henry, J., Bailey, P. \& Altgassen, M. (2013). Episodic Future Thinking in Children with Autism Spectrum Disorder. J Autism DevDisord, 43, 2558-2568. doi: 10.1007/s10803-013-1806-y

Trontel, H., Duffield, T., Bigler, E., Froehlich, A., Prigge, M., Nielsen, J.,... Lainhart, J. (2013). Fusiform Correlates of Facial Memory in Autism. Behav. Sci., 3, 348-371. doi: $10.3390 / \mathrm{bs} 3030348$

Tsatsanis, K., Noens, L., Illmann, C., Pauls, D., Volkmar, F., Schultz, R. \& Klin, A. (2011). Managing Complexity: Impact of Organization and Processing Style n Nonverbal Memory in Autism Spectrum Disorders. J Autism DevDisord, 41, 135-147. doi: 10.1007/s10803010-1139-z

Tulving, E. \& Donaldson, W. (1972). Organization of memory. New York: Academic Press.

Williams, D., Goldstein, G., Carpenter, P. \& Minshew, N. (2005). Verbal and Spatial Working Memory in Autism. Journal of Autism and Developmental Disorders, 35(6), 747- 756.doi: $10.1007 / \mathrm{s} 10803-005-0021-\mathrm{x}$

Williams, D., Boucher, J., Lind, S. \& Jarrold, C. (2013). Time-based and event- based prospective memory in autism spectrum disorder: The roles of executive function and theory of mind, and time-estimation. Journal of Autism and Developmental Disorders, 43, 1-13 http://dx.doi.org/10.1007/s10803-012-1703-9

Wheeler, M., Stuss, D. \& Tulving, E. (1997). Towards a theory of episodic memory: The frontal lobes and autonoetic consciousness. Psychological Bulletin, 121, 331-354. doi:10.1037/00332909.121.3.331.

Wilkinson, D., Best, C., Minshew, N. \& Strauss, M. (2010). Memory Awareness for Faces in Individuals with Autism. J Autism DevDisord, 40, 1371-1377 doi: 10.1007/ s10803-010-0995-x

Wojcik, D., Moulin, C. \& Souchay, C. (2013). Metamemory in Children with Autism: Exploring "Feeling-of-Knowing" in Episodic and Semantic Memory. Neuropsychology, 27(1), $19-27$.

Yerys, B., Wallace, G., Jankowski, K., Bollich, A. \& Kenworthy, L. (2011). Impaired Consonant Trigrams Test (CTT) performance relates to everyday working memory difficulties in children with Autism Spectrum Disorders. Child Neuropsychology, 17(4), 391-399. 
Yi, L., Fan, Y., Joseph, L., Huang, D., Wang, X., Li, J. \& Zou, X. (2014). Event-based prospective memory in children with autism spectrum disorder: The role of executive function. Research in Autism Spectrum Disorders, 8, 654-660. http://dx.doi.org/10.1016/j. rasd.2014.03.005

Zalla, T., Labruyere., N. \& Georgieff, N. (2013). Perceiving Goals and Actions in In dividuals with Autism Spectrum Disorders. J AutismDevDisord, 43, 2353-2365. doi: 10.1007/ s10803-013-1784-0 